\title{
Magnetic link design for a robotic laparoscopic camera
}

\author{
Massimiliano Simi, Gastone Ciuti, Selene Tognarelli, Pietro Valdastri, ${ }^{\text {a) }}$ Arianna Menciassi, \\ and Paolo Dario \\ CRIM Lab, Scuola Superiore Sant'Anna, Pisa 56127, Italy
}

(Presented 19 January 2010; received 31 October 2009; accepted 24 November 2009; published online 19 April 2010)

\begin{abstract}
The use of magnetic fields to control and steer assistive and operative devices is increasing in minimally invasive surgical applications. The design of the magnetic link between an external permanent magnet, maneuvered by an industrial robot, and a robotic laparoscopic camera was investigated in this paper, with the objective to obtain accurate positioning and steering in visualization. (c) 2010 American Institute of Physics. [doi:10.1063/1.3352581]
\end{abstract}

\section{INTRODUCTION}

Laparoscopic surgery, also called minimally invasive surgery, is a modern surgical technique in which operations in the abdomen are performed through small incisions (usually $0.5-1.5 \mathrm{~cm}$ ) as compared with larger incisions needed in traditional surgical procedures. The widespread adoption of such technique opened a new era in surgical treatments. Recently, there has been an impetus to further reduce the invasiveness of laparoscopic surgery by introducing magnetically guided devices that fit entirely inside the abdomen; thus preventing the need of a dedicated access through the skin.

From the technical standpoint, the most advanced systems exploiting magnetic fixation and positioning for laparoscopic procedures are three imaging robots reported in Refs. $1-3$. However, in these solutions the magnetic element onboard the device is not intended to move during operation. Therefore, device position and orientation are normally controlled by manually maneuvering an external permanent magnet (EPM). This results in a poor accuracy and difficult controllability of the internal device due to the exponential variability of magnetic fields with distance. To overcome this limit, the EPM can be fixed as the end effector of six degrees of freedom industrial robot (RV-3SB robot, Mitsubishi Electric, Japan) and controlled by an intuitive user interface, as proposed in Ref. 4. To further enhance precision in steering and orientation of the surgical camera, a magnetic internal mechanism (MIM) was introduced in Ref. 5.

An improved version of this device, presented in this paper, consists of a cylinder $12.7 \mathrm{~mm}$ in diameter and $32 \mathrm{~mm}$ in length. It incorporates one brushless motor, two internal permanent magnets (IPMs) and a vision module with illumination. A wired connection to an external unit guarantees signal transmission and powering, providing also an effective retrieval from the abdomen especially in case of failure. The internal mechanism consists of a motor connected to the IPM by a set of gears. When the device is immersed in an external magnetic field generated by the EPM, the IPM tends to maintain a precise alignment. Under a set of conditions, detailed below, when the motor is activated, the entire capsule rotates with it, while the IPM remains oriented according to the

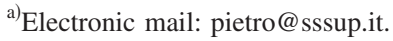

external field. Thanks to this principle of operation, the MIM enables a fine orientation of the device relatively to the surrounding tissue, without moving the EPM. The complete platform, including the robotic arm to support the EPM, is represented in Fig. 1(a).

In the present work we model and design the EPM-IPM magnetic link in order to achieve an effective magnetic interaction between the robotic maneuvered EPM and the active surgical camera. This may serve as methodological example whenever a transabdominal magnetic link is required in the design of novel surgical instrumentation.

\section{MATERIALS AND METHODS}

Based on the schematic representation reported in Fig. 1 (b), the following relations must be satisfied for proper operation:

$$
\begin{aligned}
& F_{m}>F_{w}, \\
& T_{m}>\left(T_{\text {mim }}+T_{f r}\right), \\
& T_{\text {mot }}>\left(T_{\text {mim }}+T_{f r}\right),
\end{aligned}
$$

where $\mathrm{F}_{m}$ and $\mathrm{F}_{w}$ are the EPM-IPM magnetic attraction force and the device weight force, respectively. $\mathrm{T}_{m}$ is the magnetic torque exerted by the EPM on the IPM, $\mathrm{T}_{\text {mim }}$ is the maximum torque exerted by the weight of the device on the IPM pivot, and $\mathrm{T}_{f r}$ is the adverse torque between the device and the tissue during the camera steering. Finally, $\mathrm{T}_{\text {mot }}$ is the total
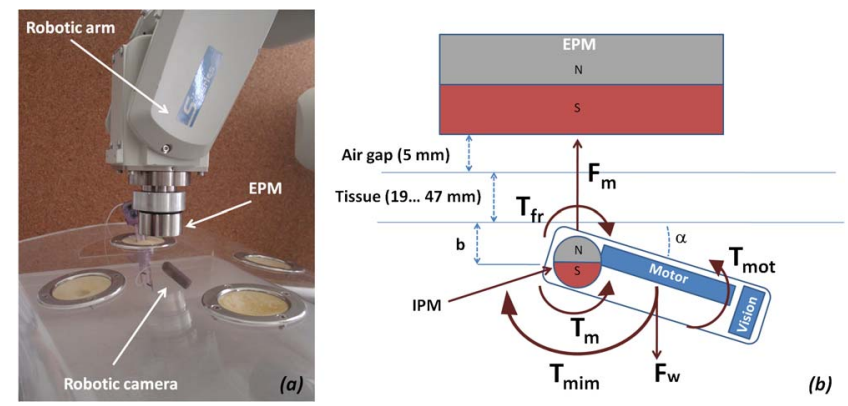

FIG. 1. (Color online) (a) The overall platform. (b) Schematic representation of the system. 
torque that the brushless motor transmits to the IPM by means of the internal mechanism.

Considering an abdominal wall thickness ranging from 19 to $47 \mathrm{~mm}$ (Ref. 6) and a constant safety air gap of $5 \mathrm{~mm}$ between the EPM and the patient skin, an IPM-EPM total distance within 24 and $52 \mathrm{~mm}$ was considered. According to the analytical background described in Refs. 7 and 8, IPM and EPM must be selected in terms of size, shape, magnetic remanence, and magnetization direction in order to fulfill the above reported relations.

\section{A. Magnetic and mechanical design of the robotic device}

As a first step, the IPM features were defined to maximize $\mathrm{F}_{m}$ and $\mathrm{T}_{m}$, while minimizing the device diameter (13 $\mathrm{mm}$ is the internal diameter of a typical trocar for laparoscopy). Two diametrically magnetized commercially available NdFeB N42 permanent magnets (KJ Magnetics, Jamison, U.S.), having a disk shape $6.35 \mathrm{~mm}$ in diameter and $3.17 \mathrm{~mm}$ in thickness, were selected. For a further maximization of the interaction force with the EPM, two custom made magnets with the same dimension, but higher remanence (i.e., N52), may be used.

In order to maximize the torque transmitted by the entire mechanical system to the IPM $\left(\mathrm{T}_{\text {mot }}\right)$, a brushless motor (SBL04-0829 Namiki precision Jewel Co. Ltd., Tokyo, Japan) was selected as best tradeoff between high stall torque $\left(\mathrm{C}_{\text {mot }}\right)(5.7 \mathrm{mNm})$ and small size $(4 \mathrm{~mm}$ in diameter and 17.5 $\mathrm{mm}$ in length). A mechanism with a low transmission ratio $(\tau)$ of 0.056 and composed by a helical gear and a worm gear connects the IPM to the motor. Based on Ref. 9, the mechanism efficiency $\left(\eta_{\text {mec }}\right)$ due to internal friction was calculated as 0.415 . In order to introduce a safety margin for the mechanism design, the half of the stall torque was adopted as the operative value. Finally, considering the magnetic interference with the electromagnetic actuator, motor efficiency $\left(\eta_{\text {mot }}\right)$ was defined to account for the performance reduction. The total torque transmitted to the internal magnets by the motor is described by the equation

$$
T_{\mathrm{mot}}=\frac{0.5 \cdot C_{\mathrm{mot}} \cdot \eta_{\mathrm{mot}} \cdot \eta_{\mathrm{mec}}}{\tau} .
$$

Once the internal magnets and all the other mechanical parts of the camera device were selected and assembled, $\mathrm{F}_{w}$ and $\mathrm{T}_{\text {mim }}$ were evaluated. The camera device is $7.7 \mathrm{~g}$ in weight; therefore a minimal magnetic attraction force larger than $75.5 \mathrm{mN}\left(\mathrm{F}_{w}\right)$ is required to lift the capsule. By knowing mass and arrangement of all device components, its center of mass $\left(8.5 \mathrm{~mm}\right.$ from the IPM pivot) was derived and $\mathrm{T}_{\mathrm{mim}}$ was analytically calculated as $0.64 \mathrm{mNm}$.

Finally, assuming a constant friction coefficient $(\mu)$ between the tissue and the camera device equal to 0.1 (Ref. 10) and a distance $(b)$ [see Fig. 1(b)] between the rotation pivot and the tissue equal to $6.35 \mathrm{~mm}$, the friction torque for the system is described by

$$
T_{f r}=\left(F_{m}-F_{w}\right) \cdot \mu \cdot b
$$

\section{B. Magnetic and dimensional design of the EPM}

Once the features of the modules on-board the device were fixed, the magnetic and dimensional properties of the EPM must be defined in order to address the requirements in term of magnetic attraction force and torque. Axial magnetization direction and cylindrical shape were purposely selected to allow the required degrees of motion and to guarantee a symmetric field along the central axis of the EPM. N52 magnetic remanence was chosen to maximize the EPMIPM attraction force and torque. The robotic arm payload (maximum weight of $3 \mathrm{Kg}$ in our case) and limitations of the workspace must also be considered during selection of an adequate EPM.

In order to refine our selection among commercially available EPM candidates already fulfilling the above requirements, finite elements method (FEM) analysis (COMSOL MULTIPHYSICS 3.4-COMSOL, Inc., Sweden) was used. Seven off-the-shelf permanent magnets having a volume ranging from 6.44 to $51.5 \mathrm{~cm}^{3}$ were considered. Magnetic attraction forces $\left(\mathrm{F}_{m}\right)$ and torques $\left(\mathrm{T}_{m}\right)$ were thus evaluated by FEM analysis between the defined IPM and the different external magnets. Magnetic flux density around the electromagnetic motor was also calculated in order to evaluate the motor efficiency $\left(\eta_{\text {mot }}\right)$. All properties of the EPM, such as magnetic permeability $\left(\mu_{0}=1.05\right)$ and different dimensions, were set into the simulation. The selected mesh consisted of about 500.000 elements with a maximum element size fixed to $1 / 100$ of the maximum geometric feature in the overall scenario. The incremental ratio of the mesh elements was fixed to 1.45 , whereas the curvature factor and the mesh curvature cut-off were chosen equal to 0.5 and 0.02 , respectively.

Based on simulation results, magnetic and physical requirements, and off-the-shelf availability, an axially magnetized disk shaped NdFeB N52 permanent magnet (KJ Magnetics, Jamison, U.S.) $50.8 \mathrm{~mm}$ in diameter, $12.7 \mathrm{~mm}$ in thickness, and $200 \mathrm{~g}$ in weight, was selected. Among the magnets that satisfied the design relations (1)-(3), the selected one represents the optimal solution in terms of magnetic force and torque.

\section{Experimental validation}

Once defined the EPM and IPM features, the simulation results, in terms of magnetic attraction force and torque, were assessed by several bench tests with a force/toque sensor (Nano17, ATI, U.S.). The IPM was fixed to the sensor and the EPM was placed as the end-effector of the robotic arm to achieve precise translational and rotational movements. The experimental setup is represented in Fig. 2.

\section{RESULTS AND DISCUSSION}

The $\mathrm{F}_{m}$ values obtained by FEM simulations were 150 and $930 \mathrm{mN}$ at EPM-IPM distances of 52 and $24 \mathrm{~mm}$, respectively. $\mathrm{T}_{m}$, considering an IPM axial rotation of $\alpha=2^{\circ}$ (minimum step of the mechanism), ranges from 1.7 to 7.1 $\mathrm{mNm}$. As required, the magnetic attraction force overcomes the weight of the device $(75.5 \mathrm{mN})$. Once $\mathrm{F}_{m}$ was known, $\mathrm{T}_{f r}$ was analytically calculated as 0.05 and $0.54 \mathrm{mNm}$ at EPM- 


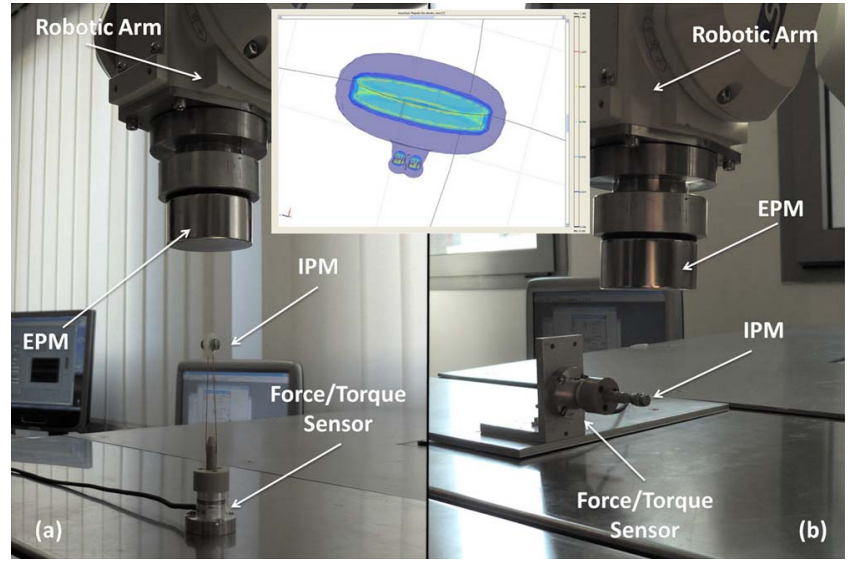

FIG. 2. (Color online) Experimental test bench for measurement of magnetic force (a) and torque (b). Inset: FEM simulation screenshot.

IPM distances of 52 and $24 \mathrm{~mm}$, respectively. By considering $\eta_{\text {mot }}$ equal to 0.9 at $5.2 \mathrm{~mm}$ and equal to 0.35 at $24 \mathrm{~mm}$, $\mathrm{T}_{\text {mot }}$ varies between 19 and $7.39 \mathrm{mNm} . \mathrm{F}_{m}, \mathrm{~T}_{m}$, and all the parameters described above confirmed Eqs. (1)-(3); thus allowing a reliable and accurate dragging and steering of the camera device. It is worth mentioning that the levels of force obtained would not cause any injury to human tissues which are in contact with the IPM (Ref. 11).

By using the experimental setup described in Sec. II, the results obtained by FEM analysis were confirmed in terms of magnetic attraction force and torque at the EPM-IPM target distances. In particular, a force of 883 and $143 \mathrm{mN}$ and a torque of 6.73 and $1.52 \mathrm{mNm}$ were obtained at EPM-IPM distances of 24 and $52 \mathrm{~mm}$, respectively. The difference between FEM and experimental values (maximum percentage difference $12 \%$ ) was mainly derived from the interference of the external magnetic field with the metal parts of the robotic arm.

In conclusion, the present work outlined the required steps to properly design a transabdominal EPM-IPM magnetic link for surgical robotic devices. This study may serve as guideline for the development of novel intracavitary robotic tools driven by magnetic fields, thus highlighting how to apply basic theoretical models to surgical applications.

\section{ACKNOWLEDGMENTS}

This material is based in part upon work supported by the European Commission in the framework of VECTOR (Contract No. EU/IST-2006-033970) and ARAKNES (Contract No. EU/IST-2008-224565) European Projects and in part by the Intelligent Microsystem Center, Korean Institute of Science and Technology (KIST), Korea.

${ }^{1}$ A. C. Lehman, K. A. Berg, J. Dumpert, N. A. Wood, A. Q. Visty, M. E. Rentschler, S. R. Platt, S. M. Farritor, and D. Oleynikov, Comput. Aided Surg. 13, 95 (2008).

${ }^{2}$ J. Cadeddu, R. Fernandez, M. Desai, R. Bergs, C. Tracy, S. J. Tang, P. Rao, M. Desai, and D. Scott, Surg. Endosc 23, 1894 (2009).

${ }^{3}$ T. Hu, P. K. Allen, N. J. Hogle, and D. L. Fowler, Int. J. Robot. Res. 28, 1373 (2009).

${ }^{4}$ G. Ciuti, P. Valdastri, A. Menciassi, and P. Dario, Robotica 28, 199 (2010).

${ }^{5}$ A. Menciassi, P. Valdastri, C. Quaglia, E. Buselli, and P. Dario, IEEE Eng. Med. Biol. Mag. 2009, 1204 (2009).

${ }^{6}$ C. Song, A. Alijani, T. Frank, G. B. Hanna, and A. Cuschieri, Surg. Endosc 20, 987 (2006).

${ }^{7}$ D. P. Arnold and J. S. Agashe, J. Phys. D 41, 1 (2008).

${ }^{8}$ F. Carpi and C. Pappone, IEEE Trans. Biomed. Eng. 56, 1482 (2009).

${ }^{9}$ M. Quirini, A. Menciassi, S. Scapellato, C. Stefanini, and P. Dario, IEEE/ ASME Trans. Mechatron. 13, 169 (2008).

${ }^{10}$ S. Loring, R. Brown, A. Gouldstone, and J. Butler, J. Biomech. 38, 2390 (2005).

${ }^{11}$ M. H. Lu, W. Yu, Q. H. Huang, Y. P. Huang, and Y. P. Zheng, IEEE Trans. Instrum. Meas. 58, 3079 (2009). 